\title{
Le rôle des revues spécialisées dans la formation des enseignants de français de l'Italie post-unitaire (1883-1915)
}

\section{Nadia Minerva}

\section{(2) OpenEdition}

\section{Journals}

Édition électronique

URL : https://journals.openedition.org/dhfles/1779

DOI : $10.4000 /$ dhfles. 1779

ISSN : 2221-4038

Éditeur

Société Internationale pour l'Histoire du Français Langue Étrangère ou Seconde

Édition imprimée

Date de publication : 1 juin 2005

ISSN : 0992-7654

\section{Référence électronique}

Nadia Minerva, «Le rôle des revues spécialisées dans la formation des enseignants de français de

I'Italie post-unitaire (1883-1915) », Documents pour l'histoire du français langue étrangère ou seconde [En ligne], 33/34 | 2005, mis en ligne le 01 janvier 2012, consulté le 27 mai 2021. URL : http://

journals.openedition.org/dhfles/1779; DOI : https://doi.org/10.4000/dhfles.1779

Ce document a été généré automatiquement le 27 mai 2021

(C) SIHFLES 


\title{
Le rôle des revues spécialisées dans la formation des enseignants de français de l'Italie post-unitaire (1883-1915)
}

\author{
Nadia Minerva
}

\footnotetext{
À la noblesse ! Un seigneur milanais, Forcé de fuir les jacobins français, Et dans ces murs fixant son domicile, Veut enseigner le langage qu'il sait bien. Il a, pour ce, méthodes singulières ; En quatre mois, écoliers, écolières, Autant que lui sauront l'italien.
}

1 En 1901, de retour d'un long séjour à l'étranger, Romeo Lovera reprend la publication du Bollettino di filologia moderna, une petite revue consacrée à l'enseignement des langues vivantes qu'il avait fondée en 1894 et dont il avait assuré la parution pendant deux ans. Ce sont les vers de Joseph-Marie Chénier qui lui viennent à l'esprit quand il évoque la situation de l'enseignement des langues vivantes dans les premières années de l'école publique (Bollettino di filologia moderna, III ${ }^{\mathrm{e}}$ année, 1, 1901:3). Le "maître italien » qui donne le titre à la nouvelle en vers de J.-M. Chénier était en effet un noble Gascon émigré en Allemagne sous la Révolution qui s'improvisa maitre de langues du jour au lendemain et se fit passer pour Milanais pour pouvoir enseigner l'italien, langue qu'il ignorait totalement. Sa supercherie ne sera jamais découverte et toute la bonne société de la ville allemande parlera gascon convaincue de parler l'italien le plus pur. Tout de suite après l'unification de l'Italie, « républicains réconciliés avec la monarchie, remarque Lovera dans le même article, prêtres défroqués, fonctionnaires des anciens gouvernements, patriotes sans titres, titrés sans argent " (c'est moi qui traduis), tous ceux qui ne purent trouver des postes plus lucratifs dans l'administration se déversèrent sur le secondaire et $\mathrm{y}$ jouèrent le rôle du Gascon ignorant. 
2 Le tableau dessiné par Lovera est très vraisemblable ; il explique pourquoi, en Italie, après l'unification (1860) l'exigence d'une formation adéquate des professeursdevient primordiale. En effet, avec la disciplinarisation du français, les classes de langues de la jeune école italienne devraient compter sur un corps enseignant important et compétent. Mais les problèmes à surmonter sont multiples. Si les institutions privées, les seules qui dispensaient l'enseignement du français avant l'unification, recrutaient surtout des natifs, on assiste maintenant à l'accès massif d'Italiens dont les compétences linguistiques laissent souvent à désirer. On est d'ailleurs conscient que la connaissance du français devrait s'accompagner d'une solide formation pédagogique. Mais où trouver des professionnels? L'université ne sera en mesure d'en fournir que plusieurs décennies plus tard et l'école de spécialisation ne commencera à fonctionner qu'en 1998 !. En l'absence de toute intervention institutionnelle, un certain nombre d'activités d'édition se mettent en place pour contribuer à la préparation de ces professeurs : manuels consacrés aux aspirants au certificat d'aptitude à l'enseignement, brochures sur les options méthodologiques, vade-mecum du futur professeur de langues, revues spécialisées. C'est sur ces dernières que je focaliserai mon attention ${ }^{1}$.

3 Leur intérêt est multiple : elles se font l'écho du débat qui anime le corps enseignant et deviennent un observatoire privilégié pour tester les compétences linguistiques et didactiques des professeurs et pour se faire une idée du niveau requis pour les élèves. Ces revues pédagogiques fournissent aussi, vu leur objectif pragmatique, un témoignage sur les véritables pratiques de classe, plus fiable que celui que peuvent apporter les programmes ministériels ou les manuels, parfois fumeux, parfois trop ambitieux. Enfin, ces périodiques sont au cœur de la problématique qui anime ce Colloque : la formation culturelle et professionnelle des maitres des langues.

\section{Les revues et leur histoire}

4 L'inventaire complet des revues et bulletins qui traitent de l'enseignement du français est encore à dresser. Pour la période 1820-1943, un répertoire de ces outils pédagogiques dirigé par Giorgio Chiosso (1997) ne signale que dix titres de périodiques partiellement ou entièrement consacrés aux langues étrangères, dont deux assez tardifs par rapport à l'essor extraordinaire de la presse d'éducation qui caractérise les années qui vont de l'Unité à la Première Guerre Mondiale : Il Cisalpino ${ }^{2}$ est publié entre 1925 et 1928 et on doit attendre 1940 pour trouver Le lingue viventi. Organo degli insegnanti di lingue. À l'état actuel de la recherche - pour les années qui nous concernent - j'ai pu repérer quatre nouveaux titres; on compte donc une douzaine de revues qui sont sans doute loin de constituer l'intégralité de la production périodique. D’ailleurs, on peut également glaner dans la presse d'éducation "généraliste" des articles concernant l'enseignement des langues vivantes. Tâche bien lourde, l'ouvrage de Chiosso comportant 1273 titres. Axés sur la problématique de l'enseignement primaire, la plupart des périodiques recensés par le chercheur turinois ne s'intéressent pas aux langues étrangères ; pourtant, un bon nombre de revues généralistes ou celles qui sont destinées au secondaire consacrent des articles aux enseignements linguistiques (langues classiques et langues vivantes). Il faudra donc se pencher, dans l'avenir, sur les divers bulletins, expression de l'associationnisme secondaire ou sur des revues comme Gymnasium, Il Caffé, La Vigilia, Nuovi doveri... 
Bornons-nous, pour cette première phase d'un travail qui s'annonce de longue durée, aux revues spécialisées ayant pour seul but l'enseignement des langues vivantes, modernes, «nouvelles » comme on disait à l'époque selon le modèle allemand. En voici une liste provisoire, fruit d'un premier recensement ${ }^{3}$ :

- Journal de la langue française et des langues en général à Naples (1839)*

- Giornale per lo insegnamento teorico-pratico della lingua francese (1874)

- La lingua francese nelle scuole secondarie d'Italia (1883-1904)

- La lingua tedesca. Periodico mensile ad uso degli insegnanti e degli studenti (1887-1905)

- L'Ape, periodico scolastico di lingua francese (1892-1913)

- Bollettino di Filologia Moderna (1894-1896, 1901-1909)*

- Cosmopolitan School (1901-1902)

- Le Parisien (1905-1915)*

- L'écho français (1910)*

- Bulletin scolaire (1912-1914)

- Mes loisirs, journal scolaire (1912-1915)

- Le Moniteur, intermédiaire des professeurs des langues vivantes (1914-1935)

La périodisation choisie se justifie donc par la concentration relevée dans les années 1880-1915, âge d'or de la presse d'éducation linguistique, qui voit naître et mourir presque toutes ces revues. Cette concentration ne se produira plus. Après 1915, la Grande Guerre et le fascisme vont freiner - et bloquer quelquefois - toute activité de promotion de la pédagogie des langues modernes et, plus en général, contraster l'associationnisme professoral. Le Moniteur restera le seul rempart des enseignements linguistiques en Italie ${ }^{6}$.

7 On aura remarqué que huit revues sur douze sont entièrement consacrées au français ; une neuvième, le Bollettino, fait une part très importante au français et une dixième, le Moniteur, est rédigée entièrement en français. Les revues les plus représentatives par leur durée, leur diffusion et le prestige de leurs auteurs sont au nombre de quatre, et nous les devons à trois directeurs: Candido Ghiotti (La lingua francese nelle scuole secondarie d'Italia), Giovanni Bonfiglio (L'Ape et Le Parisien) et Romeo Lovera (Bollettino di filologia moderna). Après la cessation du Bollettino, qu'il avait assuré pendant onze ans, Lovera fait paraître L'écho français, qui n'en est qu'une brève continuation, et une ultérieure confirmation de la vocation polyglotte de son auteur. On peut considérer ces périodiques comme des exemples réussis de presse d'éducation spécialisée de l'époque.

\section{Le panorama}

Il serait utile d'étudier le cadre de référence des revues consacrées aux langues pour les mettre en perspective avec d'autres revues italiennes de la même période et avec les revues françaises et allemandes, les plus citées dans nos périodiques. Force en est de se borner ici à quelques remarques.

9 L'effervescence éditoriale ${ }^{7}$ témoignée par le recensement de Chiosso est liée, selon l'historien de la pédagogie, à des facteurs de nature différente mais convergents: nouvelles conditions déterminées par l'institutionnalisation de l'enseignement, scolarisation de certaines disciplines, système scolaire encore instable, modalités de recrutement non homogène, naissance des associations professionnelles, sentiment généralisé de stagnation du système éducatif italien, exigence de professionnalisation 
du corps enseignant. Si ces motivations sont aussi valables pour nos revues, d'autres doivent être évoquées pour les enseignements linguistiques : ce phénomène doit être mis en rapport avec les conditions lamentables des enseignements linguistiques dans une Italie qui vient de mettre en place le système éducatif national et qui a évidemment d'autres priorités (l'alphabétisation en langue nationale de millions de dialectophones avant tout); mais il faut tenir compte aussi du mouvement de la réforme et du bouleversement méthodologique qu'il a préconisé.

10 Ce n'est pas un hasard si La lingua francese a la même date de naissance que l'Eco dell'associazione nazionale fra gli insegnanti delle scuole secondarie (1883-1887 et 1887-1890 avec un nouveau titre: Le scuole secondarie); et ce n'est pas un hasard non plus si les deux revues paraissent dans la même ville : Turin, ville où s'est constituée l'association qu'on vient de citer 8 . Entre 1860 et 1899, 82 revues s'occupent de l'enseignement secondaire (elles n'étaient que 5 jusqu'en 1859). Ces revues nous montrent que l'école secondaire italienne dans son ensemble est engagée dans divers projets de renouvellement et de réforme. On relève trois niveaux d'intervention : révision critique des méthodes et des contenus de l'enseignement qui s'accompagne d'un soutien didactique fort ; analyse des projets de réforme ; éveil d'une conscience de catégorie sur l'exemple des mouvements des pays européens par rapport auxquels on constate un grave retard. Nos périodiques se ressentent donc des conditions socio-politiques que la classe enseignante tout entière vit dans ces années ${ }^{9}$, d'où les thématiques communes à d'autres revues concernant l'enseignement secondaire. La naissance et le développement de l'associationnisme trouvent des échos forts dans les appels des directeurs de nos revues à former des associations entre les professeurs de langues vivantes ou - si on ne parvient pas à réaliser des sociétés autonomes - à adhérer à celles qui œuvrent déjà avec profit (La lingua francese, Bollettino). Le but est d'ailleurs commun : améliorer les conditions matérielles et "morales " - élever le statut des professeurs - en leur fournissant les moyens d'acquérir une formation solide (on aspire à leur assurer un niveau culturel, des compétences disciplinaires et pédagogiques), stimuler leur adhésion aux associations corporatives - dans le but précisément de rompre l'isolement où se trouvent à opérer les enseignants et de susciter la naissance d'une opinion collective sur les principaux problèmes de l'école, les règlements et les plans d'études notamment - contribuer aux débats parlementaires sur les transformations en cours. Appels et revendications que l'on trouve un peu partout dans la presse ; celle qui se consacre aux langues a aussi un autre intérêt. La lingua francese naît la même année (1883) que 17 autres revues ${ }^{10}$, dont plusieurs spécialisées comme la créature de Ghiotti et Dogliani (Il piccolo Pitagora, par exemple). C'est un phénomène caractéristique de ces années : plusieurs disciplines scolaires auront droit à des revues spécialisées et les données fournies par les index établis par Chiosso sont frappantes: une soixantaine de revues se consacrent à la gymnastique, 11 au dessin, la musique et le chant encomptent 12 , les mathématiques et la géométrie 31 ; d'autres s'occupent des travaux manuels à l'école, de la géographie, une trentaine des langues classiques... Si donc Ghiotti et compagnie partagent en partie le programme des revues destinées à l'instruction secondaire, c'est à la catégorie des outils didactiques et "disciplinaires " qu'appartiennent nos périodiques. Ils se présentent en effet comme des guides dans la forêt inextricable des méthodes, comme des supports indispensables aux activités de classe ou comme des modes d'emploi des manuels, et même - bien souvent - comme des livres du maitre de la production grammaticale ou anthologique des rédacteurs de la revue. 
11 La scène internationale confirme les données relevées pour la Péninsule : il suffit de comparer le répertoire de Chiosso avec des recensements analogues relatifs à d'autres pays européens, par exemple celui réalisé par l'équipe dirigée par Pierre Caspard (Caspard dir. 1981-1991) pour la France ou le répertoire belge de M. De Vroede (1973-1978), qui consacre un tome à la période 1817-1878, un deuxième tome à la période 1878-1895 et deux tomes à la période 1896-1914 ${ }^{11}$. D'ailleurs, les revues étrangères sont familières aux directeurs italiens qui présentent régulièrement Neophilologisches Centralblatt, Le Maître phonétique, Les quatre langues, Die Neueren Sprachen, la Revue de l'enseignement des langues vivantes... pour ne citer que les périodiques consacrés aux langues.

12 Afin de délimiter le vaste domaine évoqué, ces brèves notes ne fourniront qu'une présentation sommaire des revues répertoriées, ayant pour but l'enseignement du français. Leur analyse étant à ses débuts, ces revues ne livrent pas encore de réponses satisfaisantes; cependant les questions qu'elles posent peuvent constituer des pistes possibles de recherche.

On peut se demander s'il est légitime de considérer le Journal de la langue française et des langues en général à Naples comme une revue d'enseignement, bien que son directeur soit un professeur de français, qu'il évoque ses élèves parmi les destinataires de son périodique et qu'il soit le seul, à ma connaissance, à avoir fondé une " école normale de français " pour les futurs professeurs de cette discipline. Paru à Naples par les soins de Charles Appert, le Journal n'a, à l'état présent de mes recherches, qu'une existence annuelle. Charles Appert se propose de fournir à ses concitoyens un aperçu de ce qui se publie à Paris. On aura reconnu le périodique français source principale du maître napolitain dont il emprunte le titre : en effet, il puise du Journal de la langue française, organe de l'Institut des Langues fondé en 1837 (dont il est membre et qui compte parmi ses membres honoraires Bopp, Jacob Grimm, le Cardinal Mezzofanti, Guizot... et dont le président est Charles Nodier), plusieurs articles, notamment ceux qui ont trait à son enseignement à Naples. Il choisit, pour commencer, un compte rendu de sa propre grammaire (La lingua francese senza maestro) et sélectionne les développements qui peuvent étayer ses théories linguistiques. Le journal paraît tous les mois dans le courant de l'année 1839 et cette première "série » est surtout consacrée à la langue parlée (prononciation et prosodie) : grâce à de nombreuses interventions, le débat sur la phonétique acquiert une véritable dimension internationale. Dans sa revue, Appert publie aussi des remarques sur l'orthographe et l'étymologie, des proverbes, des avis divers, des annonces publicitaires, une rubrique bibliographique et des anecdotes pour vaincre - nous explique-t-il - l'ennui causé par les leçons de grammaire...

En 1874, du mois de juin au mois d'août, l'éditeur Paravia (le même éditeur qui publiera La lingua francese dans sa première année de vie) fait paraitre à Turin le Giornale per lo insegnamento teorico-pratico della lingua francese, cas unique de quotidien consacré à l'enseignement $d u$ français. À diffusion nationale, destiné aux professeurs du secondaire aussi bien qu'aux "amateurs" de la langue française et animé par la volonté de la diffuser, entièrement rédigé par son directeur, G. Bottero, le Giornale publie principalement des leçons de phonétique et des exercices de grammaire. Est-ce un hasard si cette revue, la première parue après l'institutionnalisation du français, est publiée à Turin, foyer depuis toujours de la langue et de la culture françaises, ville où Candido Ghiotti quelque dix ans plus tard conçoit avec l'inséparable Giustino Dogliani la première revue destinée à une vie moins éphémère? 
15 Le Journal de Charles Appert et le Giornale de Bottero se démarquent des revues des années 80 par leur durée limitée et, pour le périodique napolitain, par une diffusion locale et un décalage temporel important. Ces deux revues peuvent être considérées comme des fruits précoces, les conditions pour ce type de publication n'étant pas encore mûres. En effet, ce n'est qu'une vingtaine d'années après l'unification que les enseignements linguistiques jouiront de revues spécialisées durables; le dynamisme et la diffusion de ces dernières témoignent, certes, de l'esprit combatif et de l'engagement de leurs rédacteurs. Elles ne seraient cependant que l'expression des entreprises personnelles de quelques professeurs brillants (comme l'avaient été les journaux de Appert et de Bottero), si elles n'avaient pas joui de nouvelles conditions favorables à ce type de publication et à l'enseignement des langues vivantes, d'une stabilisation accrue du système scolaire italien et d'une conscience professionnelle qui commence à se faire jour et à se manifester.

16 Parmi les revues qui ont connu une certaine longévité, la première en date est aussi la plus durable: comme on l'a vu, Il francese nella scuole secondarie d'Italia commence à paraître en 1883 et poursuit sa publication jusqu'en 1904 ; c'est la seule de nos revues qui ait atteint vingt ans d'existence. Quelque dix ans plus tard, deux autres revues L'Ape et le Bollettino di filologia moderna s'y ajoutent. Il en résulte, d'une part une polyphonie souhaitable dans un moment de débats méthodologiques animés, d'autre part une "continuité de service» pour les professeurs de l'époque démunis de tout secours institutionnel quant à leurs besoins de formation culturelle et professionnelle. Le Parisien, séparé de ses prédécesseurs par un ultérieur décalage temporel d'environ dix ans, confirme la vocation de ces périodiques - déjà amplement prouvée, notamment par le Bollettino -à se mettre à l'écoute des mouvements de renouvellement européens et à envisager la préparation des enseignants des langues dans une dimension internationale. Je ne présenterai pas ces quatre piliers du journalisme pédagogique et didactique puisqu'ils ont bénéficié des regards de Carla Pellandra et d'Enrica Galazzi ${ }^{12}$ qui ont préconisé une analyse détaillée de ces outils didactiques généralement méconnus. Un examen comparatif rapide permet déjà des réflexions générales sur les objectifs de la presse d'enseignement, sur les conditions dans lesquelles elle s'est développée et sur son évolution, dont quelques-unes sont proposées plus bas ${ }^{13}$.

17 Le Bulletin scolaire, supplément mensuel du Bulletin de la Ligue Valdotaine dirigé par A. Réan, s'adresse aux instituteurs pour promouvoir le français dans les écoles élémentaires de la région et l'enseignement bilingue: il propose des exercices abondants, des conseils didactiques et se bat pour l'emploi du français dans les enseignements disciplinaires. La politisation du discours scolaire est inévitable: «Valdotains! - incite sa devise - [...] Pour des citoyens il n'y a rien de plus ignominieux, après la perte de la liberté, que de renoncer à sa propre langue ». Proche des autres pour son orientation pratique mais atypique pour ses destinataires, cette revue est aussi un témoignage important des efforts pour susciter une conscience linguistique plus forte.

18 Mes loisirs, qui paraît à Pinerolo (Turin) jusqu'à la guerre, est le moins systématique des périodiques examinés. Hebdomadaire à diffusion nationale dirigé par G. Balma, il se propose d'offrir aux élèves du secondaire des matériaux simples et intéressants pour leur apprendre à lire en français de façon autonome, sans l'aide du professeur. Il tient la promesse de son titre : allure décontractée, aucune érudition, grammaire absente. Il publie de la littérature pour la jeunesse (en commençant par Robinson Crusoé), des 
poèmes, des feuilletons, des articles d'actualité, des travaux d'élèves, des anecdotes et des devinettes. À signaler une section destinée aux jeunes filles. Cette revue se prête à une comparaison avec le Parisien, les situations, le lexique, les structures grammaticales des dialogues conçus par Balma et par Bonfiglio étant similaires.

La publication du Moniteur, le seul périodique de la capitale - que nous devons au seul directeur français de cette période, Théophile Fériaud - commence quand les revues de Ghiotti, Bonfiglio et Lovera ont cessé de paraître ou sont proches de leur disparition : en 1914, à quelques mois seulement de l'intervention italienne dans la guerre mondiale. Mais il semble ne se ressentir ni des événements tragiques qui frappent la Péninsule ni de la xénophobie fasciste, du moins jusqu'en 1935. Ce journal s'occupe surtout de problèmes de nature corporative et des concours pour obtenir les certificats d'aptitude à l'enseignement (jurys, sujets des épreuves, candidats...). Il comporte une partie didactique visant à la formation des futurs professeurs dont l'analyse comparative avec les leçons analogues proposée par La lingua francese ou par le Bollettino permet de se rendre compte des permanences et des transformations en cours sur les plans professionnel, institutionnel et méthodologique.

\section{Les acteurs}

\subsection{Les rédacteurs}

20 On l'a remarqué : la revue est souvent l'œuvre d'un seul artisan dévoué et tenace. Pour mieux connaître ces protagonistes de la didactique du français et relever les traits marquants de leur personnalité, il faudrait disposer de données biographiques plus sûres que celles dont nous disposons. Leur production pédagogique et scolaire nous est connue : manuels, dictionnaires, brochures méthodologiques... Mais, quelle a été leur formation, leur statut professionnel? Aucun doute sur leur versatilité: tous polyglottes, ils parlent et écrivent en français, anglais, allemand - pour Lovera on compte aussi le roumain et le grec moderne - et ils enseignent au moins deux langues vivantes. Un professeur comme Lovera, à la vie particulièrement mouvementée, mériterait qu'on suive ses péripéties à travers l'Europe, de l'Allemagne, à la Roumanie, à la France; ses expériences professionnelles à l'étranger et ses attaches avec le milieu de la Réforme ont marqué le rôle qu'il s'est donné et l'orientation du Bollettino et, plus tard, de l'Écho français: en tant qu'authentique esprit cosmopolite, observateur de la situation de l'enseignement des langues vivantes hors d'Italie, interlocuteur des réformistes les plus actifs, il veut que sa revue soit la tribune des débats du jour et le médiateur culturel et pédagogique idéal pour les maitres italiens ${ }^{14}$.

21 Cette dernière remarque permet d'aborder un thème central : les objectifs déclarés ou implicites des responsables. Ils professent unanimement l'intention de se mettre aux services des professeurs, d'en être les mentors (le mot « guide » revient très souvent). L'évolution de cette fonction, de Ghiotti à Lovera, est à mettre en relation avec les rapports plus ou moins conflictuels avec les institutions : en partant du principe que les guides des enseignants devraient être les programmes ministériels, le premier vise à les faire connaître et à moduler le plan de la revue selon les articulations des plans d'études prévus pour les différents cours (quitte à en suggérer des ajustements); Lovera se propose comme le guide d'une façon d'enseigner les langues qui contraste 
nettement avec les objectifs et les options méthodologiques proposés par les programmes ministériels.

Deux binômes dominent les déclarations programmatiques qui accompagnent, au fil des années, la vie des revues : le théorique et le pratique, l'utile et l'agréable. Quant au premier, on ne peut que souscrire aux remarques de Pierre Caspard: la presse d'éducation est «l'espace où se déploient et le point où se focalisent tout un ensemble de théories et de pratiques éducatives d'origine tant officielle que privée » (Caspard dir. 1981 : 8). Les revues italiennes, qui - pour reprendre les propres mots de Caspard offrent aux enseignants « des informations sur le contenu et l'esprit des programmes officiels, la conduite de la classe et la didactique des disciplines", dont descendre les théories et les normes (qui sont souvent trop générales, parfois obscures) dans la pratique quotidienne et dans les difficultés d'un métier qui, confronté aux aspects les plus concrets de la vie scolaire, a du mal à s'orienter parmi les théories linguistiques, les principes méthodologiques, les normes dictées par l'État, et les adaptent à une réalité qui est souvent fort éloignée du monde des théoriciens et des législateurs. Les revues sont la voix des professeurs eux-mêmes qui revendiquent leur rôle de formateurs du jeune corps enseignant et leur droit à opérer des choix autonomes dans la gestion de la vie enseignante.

Quant au deuxième binôme, il revient d'une revue à l'autre, avec une insistance particulière sur l'agréable. Les directeurs sont sensibles aux exigences d'un public scolaire jeune et démuni de toute compétence linguistique et méta-linguistique; la revue visera donc à motiver l'apprenant par des activités de plus en plus variées, faisant une part importante aux "récréations " : littérature à la portée des capacités et des goûts des adolescents, anecdotes, faits divers, jeux, devinettes, charades, nouvelles du jour.

24 La vie de la revue est scandée par de nouvelles déclarations. De nouveaux « pactes de lecture » témoignent d'une conception dynamique de la revue qui se veut souple, axée sur la modernité, capable de s'adapter à des défis toujours nouveaux.

\subsection{Les destinataires}

Le public visé dépasse souvent les frontières de l'école : les mentions aux « amateurs de la langue française" aussi bien que le courrier figurant dans quelques revues en témoignent, bien que les professeurs soient les destinataires privilégiés. Cependant - à part quelques rares cas d'intertextualité entre les revues ou de mention de quelques collègues qui se signalent par leurs écrits - les rédacteurs n'établissent pas de véritable dialogue avec leurs collègues, ils ne leur donnent presque jamais la parole. Ceux-ci restent donc de simples figurants et, si on se limite aux renseignements fournis par les revues, les multiples questions soulevées par ce mystérieux interlocuteur, presque muet, restent sans réponse. Le courrier est décevant : il n'y a souvent que les réponses $\mathrm{du}$ directeur, des réponses tellement succinctes et allusives qu'il est impossible de remonter aux problèmes suscités dans les missives des lecteurs. C'est alors par le biais des contenus de la revue et les modifications apportées, dit-on, pour satisfaire les requêtes des lecteurs qu'on peut essayer de dessiner le portrait du destinataire du périodique et de reconstruire, à travers l'offre, la demande hypothétique (de formation?, de culture ?, d'outillage didactique ?...) de ces enseignants d'autrefois. 


\section{Structure et évolution des revues} grammaire, de littérature, textes annotés, exercices souvent accompagnés d'un corrigé. Les contenus d'enseignement sont donc linguistiques (phonétique, orthographe, conversation, morphologie, syntaxe, lexique) et culturels (vie quotidienne en France et en Italie, sciences et technique, histoire, géographie, arts...). Quant à la littérature, elle se présente sous forme de textes (à signaler ceux qui semblent dépourvus de finalités didactiques précises, littérature de consommation destinée à relever le niveau culturel des professeurs ou à égayer les lectures des élèves), d'analyses de textes, d'histoire littéraire ; les genres et les siècles représentés sont variés : poésie, roman, théâtre, art épistolaire, littérature pour la jeunesse, du Moyen Âge - indispensable aux professeurs sont les leçons de grammaire historique notamment pour préparer leurs concours aux écrivains contemporains dont on se vante de publier des inédits. Les exercices sont quelquefois organisés par niveau; leur typologie est riche: versions, thèmes, questionnaires, dictées, traces de rédaction, modèles de lettres, mises en prose, compréhension, dissertation littéraire, explication de texte, traduction poétique.

Enfin, l'information bibliographique acquiert une importance toute particulière dans les visées des auteurs pour le but culturel et formatif qu'ils se proposent. Elle est multiforme: listes de publications récentes, brèves fiches signalétiques et comptes rendus et, bien sûr, avis publicitaires relatifs à la production de directeurs et collaborateurs, mais aussi de maisons d'éditions italiennes et étrangères, spécialisées en publications d'intérêt pédagogique et didactique, littéraire et linguistique. On signale des textes ayant trait à l'enseignement des langues vivantes, des œuvres littéraires ou de divulgation, on donne le sommaire d'autres revues, souvent commentées. Ces «revues des revues» sont le témoignage le plus probant de l'internationalisation souhaitée par les concepteurs des périodiques et de la circulation européenne de matériaux et d'idées. Le Bollettino cite le Maître phonétique qui publie des notices signalant les articles du Bollettino. Lovera figure aussi parmi les collaborateurs des Quatre langues. Quant à l'Allemagne, c'est un va et vient d'articles, de comptes rendus, d'annonces sur les cours d'été... 
Les rubriques naissent et meurent au fil des années selon des critères qui ne sont pas toujours clairs. Ghiotti évoque les conseils des lecteurs, mais aussi l'expérience faite les années précédentes. En revanche, les principes qui guident l'évolution du Parisien sont aisés à déceler, bien que son directeur ne les déclarent pas: cette revue - qui se démarquait des autres par sa souplesse (rubriques toujours nouvelles d'une année à l'autre, même d'un numéro à l'autre, traitement non systématique de la grammaire...) finit par se donner une structure rigide mieux adaptée à un public scolarisé et à des enseignants en quête de repères et de recettes sur lesquelles compter, sans risques, sans surprises. L'évolution de la revue, ses véritables réorientations, mais aussi les nouvelles rubriques, les nouveaux exercices sont donc à la fois un témoignage éloquent de la versatilité de cet outil didactique et du bouillonnement qui caractérisent l'enseignement des langues vivantes au carrefour des deux derniers siècles.

\section{Conclusion}

31 En conclusion de ce bref parcours, une constatation s'impose : les quelques réflexions présentées ici ne font qu'effleurer les diverses problématiques suscitées par les revues pédagogiques, les questions ouvertes étant encore trop nombreuses. En voici quelquesunes :

-Quelle a été leur diffusion réelle, au-delà des déclarations de leurs concepteurs ? Et une fois arrivées dans les divers établissements, quels en étaient les utilisateurs et comment s'en eston servi ? Leur pénétration réelle dans les milieux professionnels reste également à élucider : atteignent-elles un public plus vaste par rapport aux outils habituels (grammaires, livres de lecture...) et à la production littéraire venant de France?

- S'il est certain qu'elles ont contribué à la circulation d'idées nouvelles dans le milieu enseignant, comment mesurer l'impact des théories linguistiques et pédagogiques qu'elles ont propagées sur les professeurs de l'époque ? Ces derniers ont-ils été sensibles aux buts « nobles » (promotion culturelle et professionnelle, internationalisation...) qui ont animé les rédacteurs, ou se sont-ils limités à profiter des leçons toutes faites et des exercices avec corrigés que les revues mettaient à leur disposition?

- Quel jugement les enseignants ont-ils porté sur la qualité de l'information, sur l'efficacité des stratégies, sur la viabilité des méthodes proposées?

Si la difficulté de répondre à ces questions nous vient surtout de la rareté des sources à notre disposition, je veux cependant conclure sur une note positive : les hasards de la recherche nous mettent parfois sur des pistes fructueuses. Le Fonds Ferretti, la bibliothèque personnelle d'Angelo Ferretti, professeur de français à Reggio Emilia bibliothèque déjà célèbre parmi les habitués des colloques SIHFLES pour avoir été exploitée à plusieurs reprises par l'équipe italienne - peut nous offrir quelques bribes des réactions du corps enseignant; réactions confiées aux notes manuscrites figurant au bas de la page ou enregistrées sur de petites feuilles de cahier. Trop peu nombreuses ou trop concises, elles nous laissent souvent sur notre faim. Mais ces notes oublient de temps en temps l'habituelle discrétion et parviennent à nous communiquer les états d'âme suscités par la lecture des revues : curiosité, voire attention studieuse (Ferretti souligne, il prend des notes, il résume...) et disposition favorable aux propositions de renouveau des méthodes et des pratiques de classe d'une part et, de l'autre, méfiance à l'égard d'un directeur trop désinvolte dans l'exploitation de ses sources, perplexité vers certaines affirmations que l'on pourrait attribuer à un parti pris contestataire et 
révolutionnaire à tous prix, attitude scandalisée face aux intempérances de certains rédacteurs... Un exemple suffira : Ferretti est un lecteur averti qui a accès aux sources auxquelles puisent les rédacteurs des revues. «Che ladro [quel voleur] quel professor Lovera", s'exclame-t-il après avoir relevé les emprunts par trop audacieux du fondateur du Bollettino.

Réactions tout à fait particulières, certes, celles d'un lecteur d'exception : enseignant expert et exigeant, auteur d'un manuel et d'un essai sur les méthodes, ainsi que conteur et historien, Ferretti ne représente qu'une partie bien limitée de sa catégorie professionnelle. Sa lecture des revues est guidée par un esprit critique éveillé, nourri de vastes connaissances disciplinaires et méthodologiques dont sa bibliothèque offre un témoignage éloquent. Combien pouvait-on compter de "professeurs Ferretti » dans l'école italienne de l'époque ? De toute évidence, ce n'était pas ce type de "clientèle» qui préoccupait nos illustres directeurs pour qui la formation et la professionnalisation des enseignants des langues était un enjeu encore redoutable et incertain. À cette tâche cependant ils ont consacré une partie remarquable de leurs activités éditoriales. Qui sait avec quels résultats...

\section{Annexe ${ }^{16}$}

Le Parisien 1905-1915 (a. I, nº : oct. 1905-a. X, n²4 : juin 1915) - Caltanissetta, Tip. Panfilo Castaldi, Petrantoni.

Périodicité et description matérielle : tous les dix jours, 24 fascicules de 8 p. par an. 27 x $20 \mathrm{~cm}$. Prix : 10 cent., abonnement : Italie 2 fr., Étranger 3 fr. Présentation sur deux colonnes.

Directeur : Giovanni Bonfiglio, professeur à l'École des Mines, à l'École Technique, au Lycée de Caltanissetta. Auteur de plusieurs manuels dont: Corso completo di lingua francese, Dictionnaire des homonymes, Dizionario delle differenze sintattiche fra la lingua italiana e la francese, Letture francesi, Premiers éléments de la conversation, Corrigé des exercices ou nouveau cours.

7 Collaborateurs : A. Di Carlo, S. Messina, C. Lolli, E. Mancuso, Giulia Bonfiglio, M. Scelfo. Adele Di Carlo est auteur d'une Chrestomathie des écrivains français du Moyen Âge avec la traduction italienne en regard et Camillo Lolli de Secondo e terzo anno di lingua francese ad uso delle scuole secondarie.

Destinataires : débutants, avancés non exercés dans la conversation. A partir de la III année : public scolarisé.

Langues utilisées: français et italien pour les textes, italien pour les règles de grammaire.

Langue enseignée : français.

Objectifs : enseignement de la conversation, de la prononciation, de la grammaire. Faire apprendre la langue parlée en huit mois à travers des activités agréables.

Orientation principale : pratique : langue parlée, forme dialogique.

Bibliothèque(s) : Nationale Centrale de Florence, lacunaire.

Références bibliographiques : Galazzi 2002. 
Rubriques: «En France» (dialogues familiers), «Les phrases usuelles» (actes de parole : Pour saluer, Pour remercier, Sur la santé...), «Les occupations de Bébé », «Ce qu'il faut pour parler français", "Ce que l'on dit tous les jours", "Ce que nous voyons", "Les connaissances utiles", "La vie réelle», "Pour les dames", «Les aventures de deux étudiants », « Les leçons de la mère » (actes de paroles en contexte).

\section{Contenus d'enseignement}

-linguistiques: prononciation (règles, prononciation figurée), orthographe, conversation, morpho-syntaxe, lexique non systématiques, sous forme de notes.

-culturels : vie quotidienne (rubrique : « Tout ce que l'on dit et que l'on entend dire à Paris »), « Nouvelles du jour », sciences et techniques, littérature.

\section{Information bibliographique}

avis publicitaires : ouvrages de Bonfiglio, Di Carlo, Lolli.

\section{Littérature}

-textes: Textes courts en français avec prononciation figurée et traduction en regard: en vers (Molière, Voltaire, Lamartine, Florian, Hugo, Arnaut, Amable Testu...) ; en prose (Bernardin de Saint-Pierre, Fénelon, Zola...), lettres célèbres. Feuilleton. Extraits de pièces théâtrales.

3 Débat méthodologique / Aspects institutionnels et professionnels / Formation des enseignants : absents

\section{Évolution de la revue}

III ${ }^{e}$ année : deux nouvelles rubriques, « Théorie et pratique » : dialogues sur les thèmes habituels de la conversation courante, rangés par sujet grammatical (en italique les formes qui exemplifient la règle à enseigner), suivis de règles plus abondantes; Exercices de traduction (thème) avec corrigé. Allusion aux programmes ministériels.

IVe année : nombre croissant d'exercices scolaires - thèmes gradués. Articulation en cours élémentaire, moyen, supérieur.

$57 \quad V^{\mathrm{e}}$ année : toute la revue est divisée en deux parties, pratique et théorique.

VII ${ }^{e}$ année : nouvelle rubrique : La méthode directe (longues conversations). Disparition de la prononciation figurée. Pièces de Molière avec de riches notes linguistiques et littéraires.

\section{Bibliographie}

CHIOSSO, Giorgio (1994), «Stampa pedagogica e scolastica periodica », in Encicopedia pedagogica e scolastica, VI, Brescia, La Scuola, 1994, 11124-11153.

-----(dir.) (1997), La stampa pedagogica e scolastica in Italia - 1820-1943, Brescia,: La scuola.

GALAZZI, Enrica (1991), « La méthode phonétique pour l'enseignement du FLE en Italie à travers la lecture du Maitre Phonétique et du Bollettino di Filologia Moderna (1894-1910) », in A.M. Mandich et C. Pellandra (dir.), 277-300.

62 -----(2002), «Échos phonétiques en Sicile entre XIX et XX ${ }^{\mathrm{e}}$ siècles », in Histoire de l'enseignement du français langue étrangère ou seconde dans le bassin méditerranéen, Actes de Palerme, Documents pour l'Histoire du français langue étrangère ou seconde 28, numéro dirigé par J. Lillo, 13-31. 
CASPARD, Pierre (dir.) (1981-1991), La Presse d'éducation et d'enseignement. XVIII siècle-1940. Répertoire analytique sous la direction de Pierre Caspard, Paris, INRP, Éditions du CNRS.

MANDICH, Anna Maria et PELLANDRA, Carla (dir.) (1991), Pour une histoire de l'enseignement $d u$ français en Italie, Actes du Colloque de Parme, 14-16 juin 1990, Documents 8, sept.

PELLANDRA, Carla (1991), « Professeurs de français d'autrefois. Analyse de la revue La lingua francese nelle scuole secondarie d'Italia de 1883 à 1892 ", in A.M. Mandichet C. Pellandra (dir.), 221-228.

ROSSIGNOTTI, Giovanna (1991), « La didattica della lingua francese attraverso l'analisi del Bollettino di Filologia Moderna (1894-1895; 1901-1909)», in A.M. Mandich et C. Pellandra (dir.), 265-276.

\section{NOTES}

1. Si les études concernant l'histoire de l'enseignement du français en Italie ont porté essentiellement sur l'analyse des manuels, du cadre institutionnel et de certains protagonistes du débat méthodologique qui caractérise cette période, cet aspect de la didactique du français attend encore une reconstruction systématique, après les premières enquêtes menées lors du premier Colloque de la SIHFLES à Parme : La lingua francese nelle scuole secondarie d'Italia a été étudiée par Carla Pellandra et le Bollettino di filologia moderna par Giovanna Rossignotti. Le Bollettino a également attiré l'attention d'Enrica Galazzi qui a analysé l'impact du mouvement de la réforme sur Lovera et ses collaborateurs, les ouvertures du professeur de Salò aux études phonétiques, sa chronique, dans les pages du Bollettino, des articles du Maître phonétique et ses comptes rendus des ouvrages de Passy (cf. Mandich - Pellandra 1991 : 221-228, 265-275, 277-300).

2. Il Cisalpino est un périodique de la maison d'édition du même nom. Tout en ayant surtout un but publicitaire, il s'ouvre souvent aux contributions des spécialistes des diverses disciplines (dont les langues étrangères) et propose des articles d'intérêt didactique, des analyses de manuels et de nouvelles littéraires, artistiques et scientifiques, nationales et internationales.

3. L'ordre de présentation est chronologique ; entre parenthèses figure leur période d'existence, établie sur la base des numéros repérés. L'astérisque indique les revues non recensées par Chiosso.

4. Sa date de parution, sa nature de revue spécialisée, les noms de quelques collaborateurs qui se sont également consacrés à la didactique du français et, enfin, ses objectifs, qu'il partage avec les revues destinées aux professeurs de français font de ce périodique une base de comparaison éclairante. D'ailleurs, dans son comité de rédaction figurent des noms bien connus des chercheurs qui se sont penchés sur cette période: entre autres, nous devons à l'un de ses directeurs, G.M. Gatti, plusieurs manuels de français. Lovera, lecteur averti en tant que professeur de français et d'allemand, en fait un compte rendu très élogieux (Bollettino 1901, III, 1 : 16).

5. Cosmopolitan School constitue un cas singulier. Contrairement à ce que suggère son titre, ce périodique ne concerne pas spécialement l'enseignement des langues, mais la sténographie et un bon nombre de disciplines dispensées dans les écoles techniques. 
6. S'il n'est pas le lieu, ici, d'en élucider les raisons politiques et sociales, ce qui nous éloignerait de notre objectif - montrer l'apport des revues spécialisées à la professionnalisation des enseignants des langues - on peut rappeler de façon sommaire les conditions pénibles où se trouvèrent à opérer ces enseignants à partir de 1915. Les revues qui dépassent ce seuil sont parcourues par bien des témoignages « de guerre »; il serait utile aussi d'observer l'évolution du Moniteur après 1923.

7. 59,4\% des revues recensées par l'équipe de Chiosso paraissent entre 1880 et 1919.

8. Cf. aussi : La scuola secondaria italiana (1897-1904), La scuola media (1902-1904), La corrente (1889-1905), L'istruzione media. Organo della Federazione Insegnanti Scuola Media (1910-1925).

9. Quant à ce dernier aspect, une attention toute particulière est portée sur les questions économiques. En Italie, il y avait à l'époque deux classes d'enseignants et une double inégalité dans les salaires: on distinguait les professeurs du secondaire selon le type d'école où ils travaillaient (lycées et écoles techniques ou professionnelles), inégalité à laquelle s'en superposait une autre établie sur la base des disciplines enseignées. D'ailleurs, il faut tenir compte aussi des divers types de rapports avec l'administration et des nombreuses étapes qui précédaient la titularisation.

10. La presse périodique est destinée à s'accroître dans la décennie suivante : l'Ape voit le jour en 1892 en compagnie de 22 nouvelles revues.

11. Bijdragen tot de geschiedenis van het pedagogisch leven in België in de 19de en 20ste eeuw. De periodiken 1817-1940, 6 vol.

12. Sur La lingua francese, voir Pellandra 1991, sur le Bollettino, Rossignotti 1991 et Galazzi 1991, sur L'Ape et Le Parisien, Galazzi 2002.

13. Voir infra, les paragraphes consacrés aux « acteurs » et à la structure des revues.

14. Pour la personnalité de Ghiotti, voir l'étude déjà citée de $C$. Pellandra et, dans ces mêmes actes, la contribution de Marie-France Merger.

15. Voir, en annexe, une ébauche de fiche analysant Le Parisien.

16. La présente fiche est divisée en deux parties : la première est consacrée à une description bibliographique qui relève les éléments constitutifs de la revue, la deuxième à l'analyse des contenus.

\section{RÉSUMÉS}

En Italie, après l'unification (1860) - avec la disciplinarisation des langues étrangères - l'exigence d'une formation adéquate des professeurs devint primordiale. En effet, si les institutions privées, les seules qui dispensaient l'enseignement du français avant l'unification, embauchaient surtout des natifs, avec l'école d'État on assiste à l'accès massif d'Italiens dépourvus de compétences linguistiques et pédagogiques. Plusieurs activités éditoriales se mettent en place pour préparer ces professeurs et former de véritables professionnels, dont les revues spécialisées. Leur intérêt est multiple : écho du débat qui anime le corps enseignant, observatoire privilégié pour tester les compétences linguistiques et didactiques des professeurs, aussi bien que le niveau requis aux élèves, elles fournissent, par leur but pragmatique, un témoignage plus fiable sur les véritables pratiques de classe, par rapport aux programmes ministériels, parfois fumeux, parfois trop ambitieux, ou par rapport aux manuels qui semblent destinés à un élève modèle, idéal irréalisable de tout professeur. 
In Italy, after the unification of the country (1860) that involved the disciplinarisation of the foreign languages, the requirement of an adequate formation for teachers became central. Indeed, if the private institutions, the only ones where French was taught before the unification, especially hired natives, with the state school many Italians, often deprived of any linguistic and paedagogical competences, joined the teaching staff. Several editorial activities got in place to prepare them. Among these activities, the specialized magazines had an important role. Their interest is multiple: echo of the debate that enlivened the teaching staff, privileged observatory to test the linguistic and didactic expertises of the professors, as well as the level required to the pupils, they provide, by their pragmatic goal, a more reliable witness on the real conditions of the teaching of French, in comparison to the ministerial programs, sometimes obscure, sometimes too ambitious or to the textbooks that seem intended to a model pupil, impracticable ideal of all teachers

\section{INDEX}

Mots-clés : maître de langues, professeur, compétences linguistiques et didactiques, institutionnalisation, formation, disciplinarisation, Italie, XIXe siècle

Keywords : language schoolmaster, teacher, institutionnalisation, disciplinarisation, linguistic and paedagogical competences, Italy, XIXth century

\section{AUTEUR}

NADIA MINERVA

Université de Bologne 